\title{
Bedeutung und Potenziale der Materialflusskostenrechnung für die Steigerung der Ressourceneffizienz in energieintensiven Unternehmen
}

\author{
Milan Topic und Karin Tschiggerl \\ Lehrstuhl Wirtschafts- und Betriebswissenschaften, Leoben, Österreich
}

Eingegangen 6. November 2018; angenommen 26. November 2018; online publiziert 10. Januar 2019

\begin{abstract}
Zusammenfassung: Steigende Materialkosten, neue (Umwelt-)Vorschriften und die Sorge um die Energiesicherheit treiben Bemühungen, die industrielle Ressourceneffizienz in der Europäischen Union zu erhöhen, stetig voran. Besonders energieintensive Industrien sind damit konfrontiert, die Ressourceneffizienz in ihren Prozessen zu implementieren und zu verbessern, da diese Kosten bereits mehr als $45 \%$ ihrer Gesamtkosten ausmachen. Um das Unternehmen im betriebswirtschaftlichen Bereich zu unterstützen, wird die Materialflusskostenrechnung als ein strategisches Instrument verwendet. Die Materialflusskostenrechnung (MFKR) unterstützt das Unternehmen dabei, indem sie physische und finanzielle Daten verknüpft und Transparenz in Material- und Energieströmen sowie deren Kosten bringt. Das Ziel dieses Artikels ist es, die Bedeutung und mögliche Potenziale der MFKR für die Steigerung der Ressourceneffizienz in energieintensiven Unternehmen zu veranschaulichen und zu diskutieren.
\end{abstract}

Schlüsselwörter: Ressourceneffizienz, Materialflusskostenrechnung, Gießerei, Effizienz

Importance and Potential of Material Flow Cost Accounting to Increase the Resource Efficiency in Energyintensive Companies

Abstract: Rising material costs, new (environmental) regulations, and concerns about energy security are key issues to increase the industrial resource efficiency in the European Union. Particularly energy-intensive industries are faced with the task of implementing and improving resource efficiency in their processes as these costs already account for more than $45 \%$ of their total costs. To support the company in business management, material flow cost

Dr. M. Topic ( $\square)$

Lehrstuhl Wirtschafts- und Betriebswissenschaften,

Peter Tunner Straße 25-27,

8700 Leoben, Österreich

milan.topic@unileoben.ac.at accounting is used as a strategic tool. The material flow accounting (MFKR) helps a company by linking physical and financial data and shows transparency in material and energy flows as well as their costs. The aim of this article is to illustrate and discuss the importance and potential of MFKR for increasing resource efficiency in energy-intensive companies.

Keywords: Resource efficiency, Material flow accounting, Foundry, Efficiency

\section{Einleitung}

Die Ressourceneffizienz wird für produzierende Unternehmen zu einem zunehmend wichtigen Thema. Vor allem in den energieintensiven Industrien stellen die Materialund Energiekosten mit einem Anteil von über $45 \%$ einen erheblichen Kostenfaktor dar, der durch die Preisentwicklung deutliche Steigerungen aufweist. Gleichzeitig sinken die Personalkosten anteilmäßig. Dementsprechend liegt der Fokus der Kostenreduzierung heute nicht nur im Personalbereich, sondern auch auf dem Materialeinsatz [1]. Die Vorteile für produzierende Unternehmen sind vielseitig, einerseits wird der Wettbewerbsvorteil gegenüber Konkurrenten gesichert, da jede prozentuelle Ressourceneinsparung wesentliche Kostenvorteile und eine mögliche Verbesserung der Marktposition bedeutet. Anderseits hilft die Ressourceneffizienz dabei, neue innovative Lösungen (u. a technisch, organisatorisch, finanziell und mehr) zu finden, und liefert einen wichtigen Beitrag zur Nachhaltigkeit durch die Reduktion von Umweltwirkungen [1-3]. Trotzdem gibt es in der Priorisierung bzw. Identifikation dieses Themas innerhalb der Unternehmen Herausforderungen, da für die meisten existenzielle Fragen, wie die Unternehmensfinanzierung, Personalmangel oder Standortfragen, von größerer Bedeutung sind [4]. Des Weiteren entstehen in Unternehmen auch Unklarheiten, ob eine verbesserte Ressourceneffizienz zu Kosteneinsparungen führen kann 
Abb. 1: PDCA-Kreislauffür MFKR-Implementierung [6]

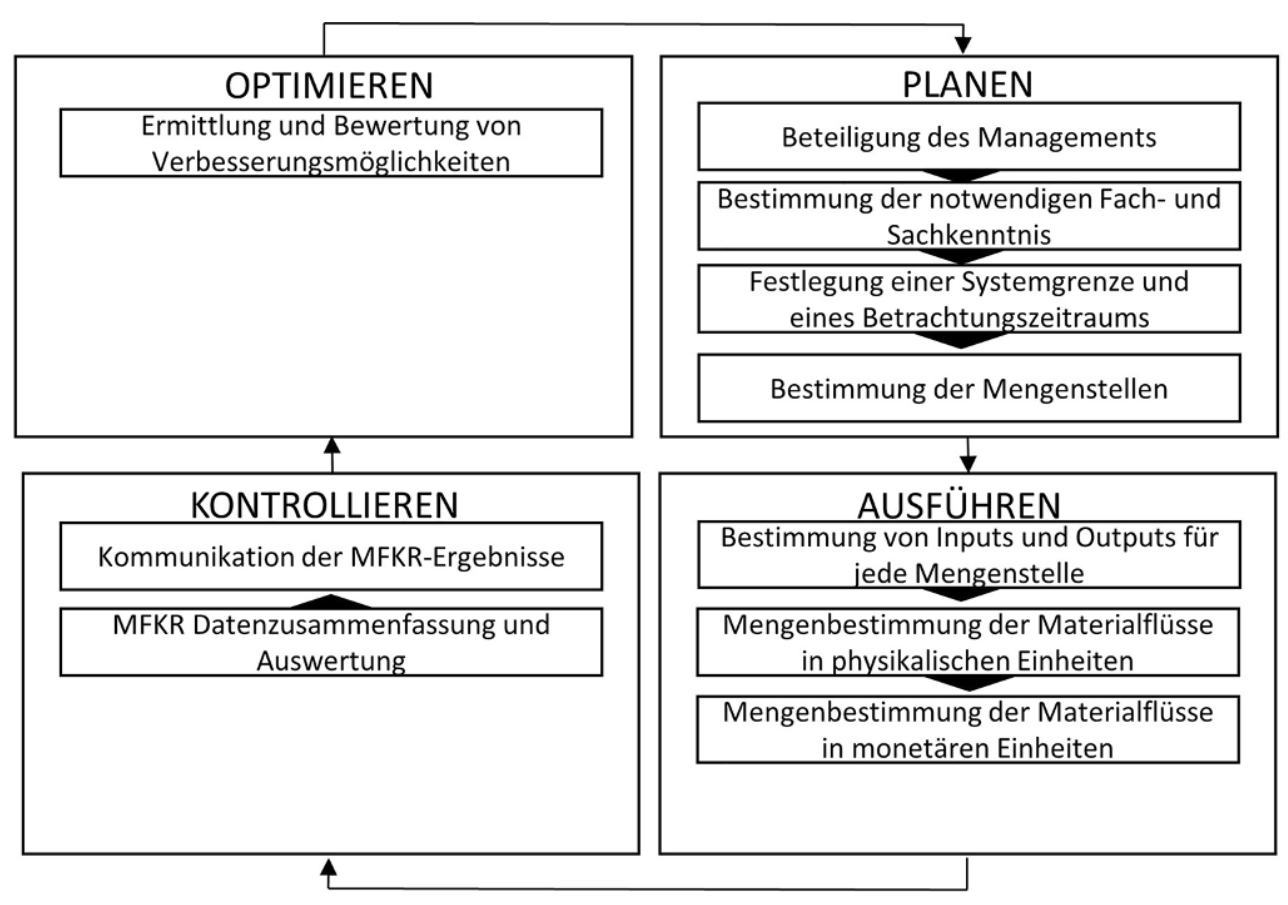

sowie wie man Material- und Energieverbräuche systematisch erfasst, Verschwendungen analysiert und bewertet und dementsprechend Maßnahmen ableitet [1, 5]. Um diese Herausforderungen zu minimieren, existieren in der Praxis verschiedene Analysemethoden und Instrumente. Diese umfassen einfache Prozessanalysen, wie ABC-AnaIyse, detaillierten Input-Outbilanzen bzw. Stoffstromanalysen, bis zu spezifischen Analysen, wie Ökobilanzierungen, Wertstromanalysen oder Materialflusskostenrechnung [1]. In diesem Beitrag wird auf die nach ISO 14051 definierte Materialflusskostenrechnung (MFKR) näher eingegangen und ihre Bedeutung und Potenziale für die Steigerung der Ressourceneffizienz in energieintensiven Unternehmen erläutert.

\section{Materialflusskostenrechnung nach ISO 14051}

Die Materialflusskostenrechnung, welche mit der ISO 14051 standardisiert wurde, ist ein Managementinstrument, das auf der Analyse von Stoffströmen basiert und zu den prozessorientierten Umweltkostenrechnungssystemen gezählt wird [6]. Sie unterstützt Unternehmen, die potenziellen umweltbezogenen und monetären Auswirkungen des Material- und Energieverbrauchs besser zu verstehen und in weiterer Folge effizient zu nutzen. Sie zeigt im Detail, wohin sich die Materialien bewegen, wo sie gelagert werden und wie viel Energie in den Prozess-(Mengen)stellen eingesetzt wurden. Dabei leistet sie folgendes [6]:

- Verbesserung der Transparenz der Material- und Energieflüsse.

- Entscheidungsunterstützung.
- Verbesserung der Koordination der Material- und Energieverbräuche innerhalb des Unternehmens.

- Verbesserung der Koordination der Material- und Energieverbräuche.

Die Methodik der MFKR verknüpft physikalische Daten der Materialmenge (z.B. Masse [kg] oder eingesetzte Energie [kWh]) mit finanziellen Daten und stellt sie übersichtlich in einem Materialflussmodell dar. Dabei werden drei verschiedene Kostenarten ermittelt: Materialkosten, Systemkosten und Abfallmanagementkosten. Energiekosten werden entweder als vierte ergänzende Kostenart angeführt, können aber auch zu den Materialkosten gezählt werden [4]. Ein weiterer Grundsatz, der Einsatz von einheitlichen Maßeinheiten, stellt sicher, dass die physikalischen Daten vergleichbar sind und die Fehlerfreiheit und Vollständigkeit gewährleistet wird. Die Aufwendungen für Stoffverluste werden nicht, wie in der klassischen Kostenrechnung, den Produkten zugerechnet, sondern gesondert erfasst. Die entstandenen Aufwendungen werden möglichst genau veranschlagt und dem Materialverlust zugerechnet. Mit dieser Methodik werden die genaueren und oft "versteckten" Kosten ersichtlich, denn die Kosten der Verluste sind in der Praxis deutlich höher.

Für die Implementierung einer MFKR schlägt die ISO 14051 den PDCA-Zyklus nach Deming vor, der in Abb. 1 dargestellt ist. Das Hauptaugenmerk dieser Methode liegt darin, Prozesse kontinuierlich mit dem Ziel einer Qualitätssteigerung und Kostenminimierung zu verbessern. Die Ergebnisse einer MFRK zeigen eine detaillierte Auflistung der Kosten und Verluste in tabellarischer und grafischer Form. Diese sollten in weitere Folge durch die Überprüfung jener Parameter, die besonders relevant für eine Entscheidung bzw. Auswahl einer Option in Form von Szenarien sind, interpretiert werden. Daraus können Maßnahmen abgeleitet 
Abb. 2: Direkte und indirekte Allokation von Energiedaten

Energiekosten pro Produkt - indirekte Zuteilung

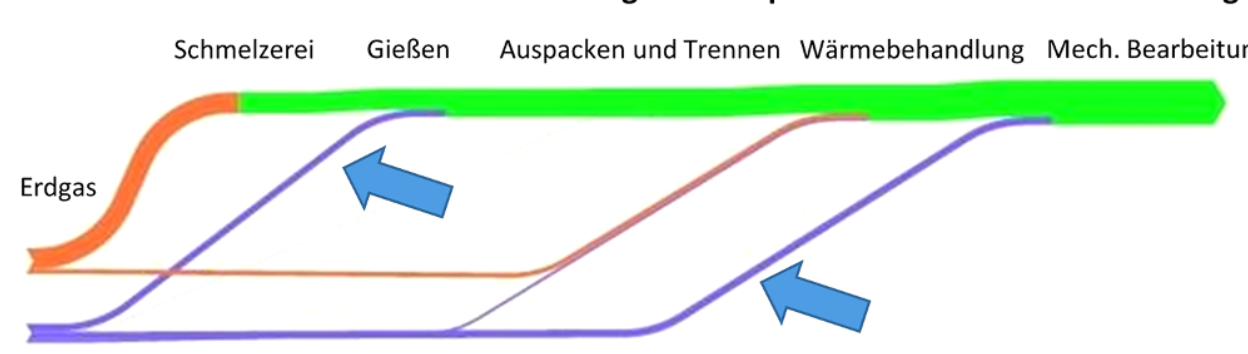

Strom

Energiekosten pro Produkt - direkte Berechnung

Schmelzerei Gießen Auspacken und Trennen Wärmebehandlung Mech. Bearbeitung

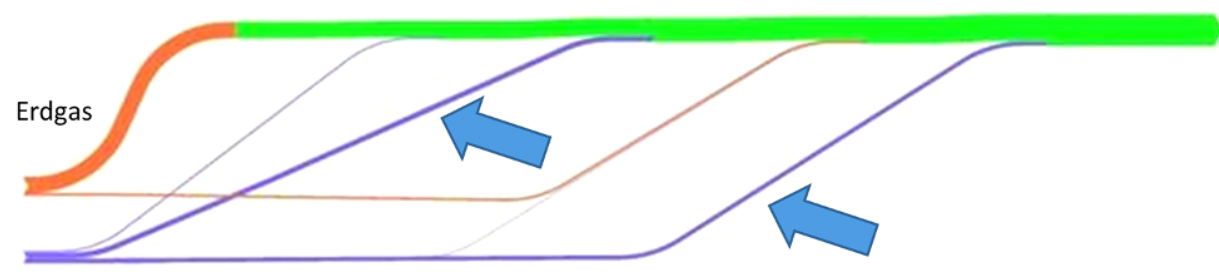

Strom

werden und anschließend vom Management für Verbesserungen der kosten- und umweltbezogenen Leistungsfähigkeit verwendet werden. Die Maßnahmen können den effizienteren Einsatz von Materialien, den Ablauf von Prozessen, Austausch von Energiequellen, Veränderung der Kreislaufmaterialquote betreffen oder verstärkte Forschungs- und Entwicklungsarbeit anstoßen [7].

Die Durchführung der MFKR kann durch mehrere Softwarelösungen unterstützt werden. Beispielhaft sind die kostenpflichtigen Softwaretools der Umberto Produktfamilie von Firma ifu Hamburg zu nennen, welche seit Jahren von Praktiker und Forscher für die Stoff- und Energieflussanalyse mit integrierter Kostenrechnung und für die Betrachtung von Umweltauswirkungen eingesetzt werden [8]. Ebenso stellt das VDI-Zentrum für Ressourceneffizienz einen kostenlosen Onlinerechner auf seiner Homepage zur Verfügung. Dieser MFKR-Rechner unterstützt den Anwender durch eine strukturierte Eingabemöglichkeit und eine grafische Darstellung des Produktionsprozesses [9].

\section{Potenziale von Materialflusskosten- rechnung für das energieintensive Unternehmen}

Die Steigerung der Ressourceneffizienz in energieintensiven Unternehmen ist ein wichtiger Faktor für ihre Wettbewerbsfähigkeit. Der erste Schritt, um das Unternehmen ressourceneffizienter zu machen, besteht darin, die eigenen Verbräuche zu kennen und vorhandene Einsparpotenziale zu erfassen. Dazu ist die Materialflusskostenrechnung ein geeignetes Instrument und Hilfsmittel, da die Ineffizienzen in den Produktionssystemen nicht nur mit Material- und Energieverlusten verbunden sind, sondern immer auch mit Kosten, die über das innerbetriebliche Abfallwirt- schaftssystem hinausgehen. Die Anwendung dieses Instruments und die daraus gewonnen Erkenntnisse bilden eine gute Basis für die Entwicklung passender Maßnahmen für die Verbesserung der Leistungsfähigkeit. Außerdem sind für das Unternehmen folgende Potenziale von größeren Bedeutung [10]:

- Identifizierung von ökonomischen und ökologischen "Hot-Spots“ innerhalb des Unternehmens zur Verbesserung der Ressourceneffizienz.

- Verbesserung der Ressourceneffizienz und Reduzierung der direkten Kosten.

- Transparenz in den Produktionskosten.

- Liefert die Anreize für Innovation.

- Unterstützung bei Produktverbesserung und Produktdesign.

- Förderung von innerbetrieblichen Recycling.

- Verbessert die innerbetriebliche Kommunikation.

- Verbessert das Controlling und die Steuerung von Entscheidungen.

- Wissensmanagement.

In weiterer Folge liefern die von der MFRK bereitgestellten Daten auch eine Grundlage für die Entwicklung weiterer Umweltmanagementaktivitäten, zu denen die Investitionsbewertung, die Umweltverträglichkeitsprüfung sowie kurz- und langfristige Umweltbudgetierung gehören können [10].

Der Hauptnutzen von MFKR für energieintensive Unternehmen besteht darin, Möglichkeiten zur Verbesserung von Ineffizienzen bei Prozessen in der Produktion oder Produkten zu identifizieren. Die Genauigkeit der Resultate ist abhängig von der Qualität der erhobenen Daten für Inputs und Outputs. Um die Analysegenauigkeit und Aussagekraft der MFKR zu erhalten/erhöhen, sollten jegliche 


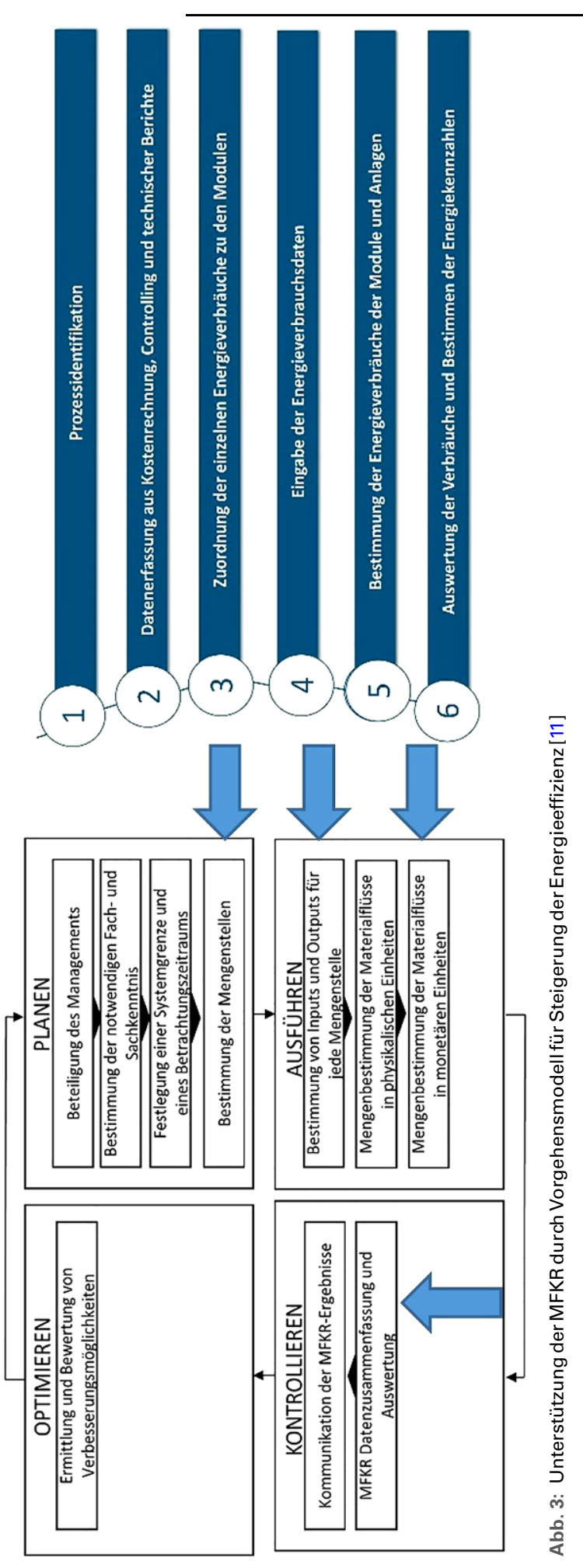


Kosten aus den Daten, die für einzelne Mengenstellen und einzelne Materialflüsse verfügbar sind, berechnet und direkt zugeteilt werden [6]. Während für viele Unternehmen das Wissen über den gesamten Material- und Energieverbrauch vorhanden ist, lassen sich diese oft nicht den konkreten verursachten Produkten und Prozessen zuordnen. Kosten können dann indirekt den Objekten (Produkten/ Prozesse) mithilfe von Verteilungsschlüsseln zugerechnet werden. In der MFKR umfasst der Begriff „Objekte“: Mengenstellen, Produkte, Materialverluste und Prozesse. Um die Analysegenauigkeit und Aussagekraft der MFKR zu erhalten/erhöhen, sollte die direkte Zurechnung der Kosten in jedem Fall der indirekten Zuordnung vorgezogen werden. Die Wichtigkeit der genaueren Allokation von Energiedaten ist in Abb. 2 illustriert. Dabei sind einerseits Energieverbräuche pro hergestelltem Produkt in einer GieBerei dargestellt, die durch indirekte Zuteilung von Daten aus dem Controlling stammen. Anderseits repräsentiert das untere Sankey-Diagramm den tatsächlichen Energieverbrauch, der aufgrund thermodynamischer Beziehungen und physikalischer Eigenschaften berechnet wurde. Die Unterschiede zwischen diesen beiden Betrachtungen sind vor allem in den Bereichen Gießen und mechanische Bearbeitung sichtbar, welche in weiterer Folge eine falsche Datenbasis für die Maßnahmenableitung zur Steigerung der Energieeffizienz liefern konnten.

Im Rahmen des Forschungsprojektes „EnEffGieß“ konnte das Nachhaltigkeitsteam des WBW ein praktisches Vorgehensmodell zur Steigerung der Energieeffizienz am Beispiel der Gießerei-Industrie entwickeln und mit den Ergebnissen die Entscheidungsgrundlagen zur Maßnahmendefinition im Bereich Material- und Energiemanagement bilden [11]. Das Vorgehensmodell "Quick Check Energie-Effiziente Gießerei“ ermöglicht in sechs Schritten ein effizientes, prozessorientiertes und transparentes Energiecontrolling und liefert dem Unternehmen rasch einen Überblick über Energieverbräuche und -kosten auf Unternehmens-, Prozessund Produktebene. Der Ansatz erlaubt die Identifizierung und transparente Darstellung/Zuteilung von Material- und Energieflüssen im Unternehmen und bereitet aussagekräftige Energie-Kennzahlen vor, die in Managementsystemen und Audits gefordert werden. Diese Methodik unterstützt die Durchführung der MFKR in mehreren Schritten, z. B. bei der Bestimmung der Mengenstellen, der Erstellung von Input und Output Bilanzen und deren Quantifizierung und bei der Monetarisierung sowie bei der Auswertung und Kommunikation (siehe Abb. 3).

Der Mehrwert von MFKR in energieintensiven Unternehmen liegt des Weiteren in der Verbesserung der Ressourceneffizienz und Förderung des internen Recyclings (z. B. Behandlung interner Kreisläufe). Interne Kreisläufe, die in den meisten Branchen vorkommen, benötigen zusätzliche Energie und verursachen eine Verschwendung von Systemkosten. Dem Nutzen des internen Recyclings steht ein ökonomischer und ökologischer Aufwand gegenüber, der abgewogen werden muss. Es gilt, den Fokus des Unternehmens auf die Reduktion der Materialströme zu legen. Die Reduktion soll zuerst durch einen sparsamen Umgang mit Ressourcen oder aber durch Vermeiden von Ausschuss herbeigeführt werden. Erst als zweite Möglichkeit soll das interne Recycling zur Reduktion der Stoffströme in Erwägung gezogen werden. Bei dem Vorteil, den die interne Kreislaufführung - ohne Zweifel - gegenüber der externen Entsorgung hat, wird der Aufwand jedoch von den meisten Unternehmen unterschätzt [12]. Die MFKR kann hierbei das Unternehmen unterstützen, das Einsparpotenzial beim internen Recycling aufzuzeigen. Dabei sind zwei Verfahren möglich, entweder durch Szenarienvergleich oder durch Ausschleusen der Kreislaufkosten. Beim Szenarienvergleich wird der rein hypothetische Fall einer Minimierung der Materialrückläufe auf Null abgebildet. Bei der zweiten Variante hat der Produktionsprozess als Output ein Kuppelprodukt, welches aus Produkt und Reststoff besteht. Die Allokation des Aufwands erfolgt massebezogen auf das Produkt bzw. den Reststoff. Dabei wird die Bewertung des durch die Aufbereitung wiedergewonnen Rohstoffs zum ursprünglichen Rohstoffpreis vorgenommen. Das Einsparpotenzial setzt sich daher aus dem internen Verrechnungspreis abzüglich des Rohstoffpreises zusammen [12].

Zur Veranschaulichung und praktischen Darstellung von Potenzialen der MFKR in den energieintensiven Unternehmen wird in Abb. 4 in Form eines Sankey-Diagrammes ein Modelunternehmen vorgestellt, das auf Basis der im Projekt EnEffGieß beteiligten Firmenpartner und den dort erhobenen Daten entwickelt wurde. Die detaillierte Analyse zeigte die ökonomischen und ökologischen „Hot-Spots“ zur Verbesserung der Ressourceneffizienz innerhalb des Unternehmens deutlich und bezifferte die "theoretischen" Energie- und Materialverluste mit ca. $10 \%$ der gesamten Energie- und Materialkosten.

\section{Zusammenfassung und Ausblick}

Die Methodik der MFKR erweist sich als geeignetes Mittel, um den Ist-Zustand des energieintensiven Unternehmens darzustellen und eine Reihe von Potenzialen für die Steigerung der Ressourceneffizienz zu identifizieren. Die durchgängige Betrachtung der Flüsse und Modellierung als Sankey-Diagramm fördert die Transparenz. Das Ausweisen der Kosten für die Verlustströme schließt eine Lücke, die die klassische Kostenrechnung hinterlässt und liefert entscheidungsrelevante Informationen für das Management zur Umsetzung von Effizienzmaßnahmen. Die Verwendung der Materialflusskostenrechnung zur Berechnung von Einsparpotenzialen muss jedoch eingeschränkt werden. Durch die Annahme eines linearen Zusammenhangs zwischen dem Input und Output wird die Realität oft stark vereinfacht. Gerade in energieintensiven Unternehmen mit hochkomplexen Prozessen kann dies problematisch sein. Für energieintensive Unternehmen sind zum einen hohe Rohstoff-, Energie-, Wartungs- und Instandhaltungskosten für Anlagen und Aggregate im Betrieb relevant. Insbesondere deren Folgekosten in Hinblick auf (Energie- und Material-) Effizienz wirken sich auf die Herstellkosten von Endprodukten aus. Zum anderen ist aus Kundensicht z. B. der Wirkungsgrad von Endprodukten, welcher von Endprodukten direkt oder indirekt beeinflusst wird, ausschlaggebend für Folgekosten, welche während des Betriebs bzw. 


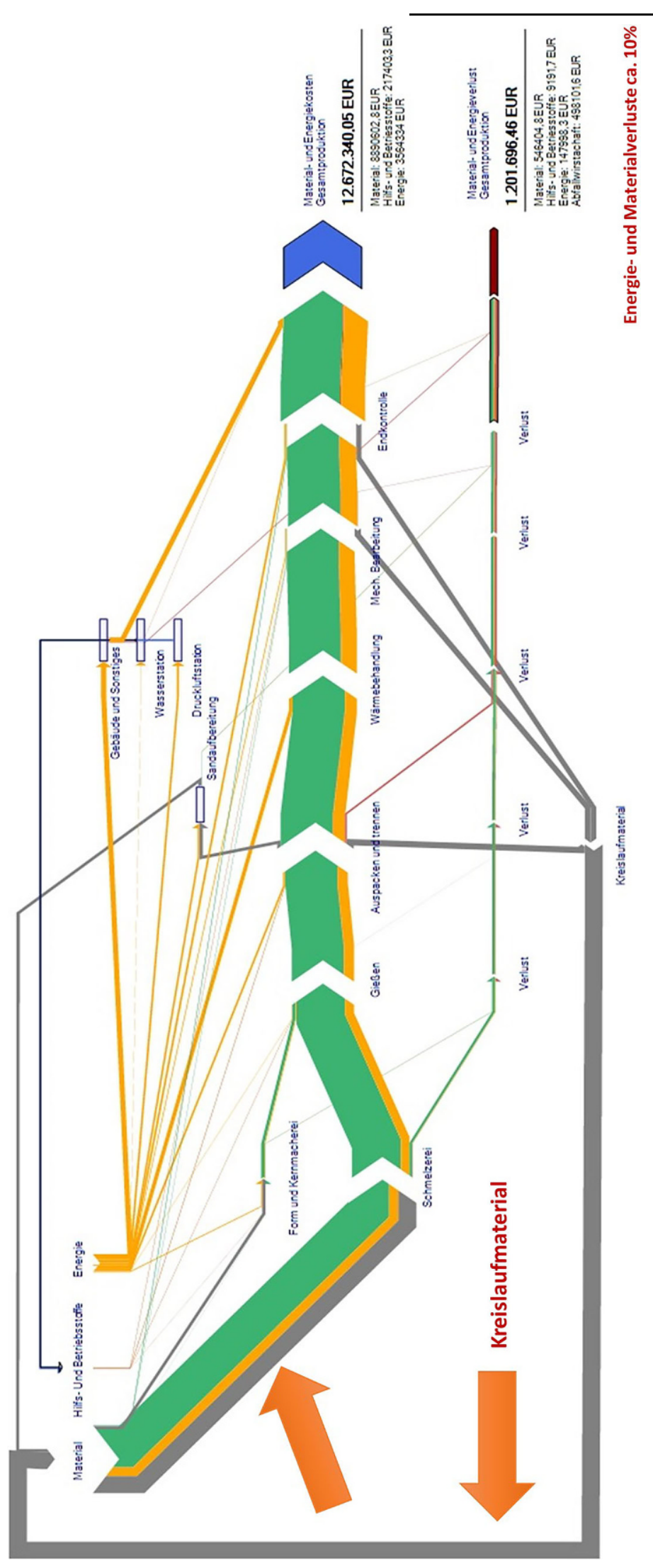

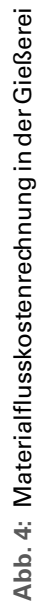


der Nutzungsphase entstehen [2]. Um das volle Potenzial der MFKR auszuschöpfen, ist die Ausweitung der MFKR auf die gesamte Wertschöpfungskette notwendig [13]. Des Weiteren liefert die zunehmende Digitalisierung der Produktion im Rahmen von „Industrie 4.0“ zahlreiche neue Impulse zur Steigerung der Ressourceneffizienz. Dabei können die Elemente von Industrie 4.0 (wie Big Data, Data-Mining, Cloud Computing usw.) in den meisten Fällen in die MFKR sehr gut integriert werden. Zum Beispiel stellt die automatisierte Erfassung aller Stoffflüsse und damit auch aller Inputs und Outputs der Mengenstellen eine enorme Erleichterung bei der Informationserhebung dar. Die Zuweisung der Kosten kann anschließend erfolgen oder mithilfe einer vorherigen Aufschlüsselung der Material-, Energie- und Systemkosten gleich miteinbezogen werden. Werden die Produkte selbst mit Informations- und Kommunikationstechnologien ausgerüstet und zu smarten Produkten gemacht, so ergibt sich dadurch die Möglichkeit ein Produkt entlang seiner gesamten Nutzungsphase zu analysieren und Rückschlüsse auf seine Lebensdauer und optimale Nutzung zu treffen.

Funding. Open access funding provided by Montanuniversität Leoben

Open Access Dieser Artikel wird unter der Creative Commons Namensnennung 4.0 International Lizenz (http://creativecommons.org/licenses/ by/4.0/deed.de) veröffentlicht, welche die Nutzung, Vervielfältigung,

Bearbeitung, Verbreitung und Wiedergabe in jeglichem Medium und Format erlaubt, sofern Sie den/die ursprünglichen Autor(en) und die Quelle ordnungsgemäß nennen, einen Link zur Creative Commons Lizenz beifügen und angeben, ob Änderungen vorgenommen wurden.

\section{Literatur}

1. Schmidt, M.; Spieth H.; Bauer, J.; Haubach, C.: 100 Betriebe für Ressourceneffizienz - Band 1, Berlin: Springer Spektrum, 2017

2. Tschiggerl, K.; Topic, M.; Doschek, K.; Rauter, M., Biedermann, H.; Raupenstrauch, H.: Gießereiprodukte und ihr Mehrwert bei Le- benszyklusbetrachtungen, Gießerei Rundschau, 62 (2015), Nr. 7/8, S. 187-194

3. Topic, M.; Tschiggerl, K.; Biedermann, H.: Lebenszyklusanalysen in der Gießerei-Industrie und deren Bedeutung im Rahmen operativer und strategischer Entscheidungen, Gießerei, 104 (2017), Nr. 7, S. 30-40

4. Engelmann, T.; Liedtke, C.; Rohn, H.; Bowry, J.: Nachhaltiges Wirtschaften im Mittelstande - Möglichkeiten zur Steigerung der Ressourceneffizienz in kleinen und mittleren Unternehmen, Bonn: Abteilung Wirtschafts- und Sozialpolitik der Friedrich-Ebert-Stiftung, 2013

5. Erhardt, R.; Pastewski, N.: Relevanz der Ressourceneffizienz für Unternehmen des produzierenden Gewerbes, Stuttgart: Fraunhofer IAO, 2010

6. ÖNORM EN ISO 14051:2011-11: Umweltmanagement- Materialflusskostenrechnung - Allgemeine Rahmenbedingungen, Wien: Österreichisches Norminstitut, 2011

7. Schmidt, M.: Material Flow Cost Accounting in der produzierenden Industrie, in von Huaff M.; Isenmann G.; Müller-Chirst. G (Hrsg.): Industrial Ecology Management, Karlsruhe: Springer, 2012

8. Ifu Hamburg https://www.ifu.com/fileadmin/user_upload/umberto/ Effizienz-Software/Umberto_NXT_MFCA/Dateien/Umberto_NTX MFCA_Software_fuer_Materialflusskostenrechnung.pdf (28.09.2018)

9. VDI-Zentrum für Ressourceneffizienz https://www.ressourcedeutschland.de/instrumente/kostenrechner-tool/ (28.09.2018)

10. Christ, K.; Burritt, R.: Material flow cost accounting: a review and agenda for future research, Journal of Cleaner Production, 108 (2015), pp 1378-1389

11. Topic, M.; Tschiggerl, K.: Integriertes Bewertungsmodell für energieeffiziente Gießereiprodukte, in Pautmeier, M. (Hrsg.): Praxis Energiemanagement, Energieeffizienz steigern, Kosten senken, 18 Aktualisierung, Köln: TÜV Rheinland, 2017

12. Viere, T.; Möller, A.; Schmidt, A.: Methodische Behandlung interner Materialkreisläufe in der Materialflusskostenrechnung. In: uwf, Vol. 18 (2010), S. 203-208

13. Schrack, D.: Die Materialflusskostenrechnung in der Lieferkette: Mengen- und Kostenwirkungen auf vor- und nachgelagerte Stufen und Entwicklung eines lebenswegbezogenen Kennzahlensystems. In: Prammer, H.-K.(Hrsg.): Ressourceneffizientes Wirtschaften: Management der Materialflüsse als Herausforderung für Politik und Unternehmen. Wiesbaden: Springer Gabler Verlag, 2014 